\title{
Tuberculosis of Liver: A Case Report
}

\author{
Author \\ Dr Banamali Nath \\ Corresponding Author \\ Dr Banamali Nath \\ MS, MNAMS, FAIS (Surgery), R.D. Gardi Medical College, Surasa, Ujjain, M.P. 456001 \\ Email: dr.banamalinathnath42@gmail.com, Mobile-9435064849
}

\begin{abstract}
Tuberculosis of liver is a rare disease. Pain in right hypochondrium and hepatomegaly are the main symptom and sign.A case of tuberculosis of liver is reported, that presented with hepatomegaly and pain in the right upper abdomen. Diagnosis of hepatic tuberculosis was made after laparotomy and liver histology. The patient recovered completely after a course of antitubercular therapy

Keywords- Tuberculosis; hepatomegaly; liver biopsy; antitubercular therapy
\end{abstract}

\section{Introduction}

Tuberculosis of liver is an uncommon disease. Liver involvement may occur in untreated or inadequately treated case of pulmonary tuberculosis. Clinical pictures may be variable. Common symptom and signs include pain in right upper abdomen and hepatomegaly. Laboratory investigations and imaging methods are often normal. Diagnosis of tuberculosis of liver rests on histology of the lesion. A rare form of tuberculosis of liver is presented here.

\section{Case Report}

The patient was a 41 years old male with complaints of distension and pain of the right upper abdomen for last 8 months. The pain was dull aching in nature and there was no aggravating or relieving factor in relation to food. On examination the patient was pale and a lump of 3"x2" size was palpable in the right hypochondrium arising from the right lobe of the liver, firm in consistency and with irregular surface and ill defined margins. Examination of chest and C.V.S were found to be normal.

Prior to hospitalisation the patient received a course of antiamoebic drugs for two weeks without any improvement. Patient's investigation showed, $\mathrm{Hb}-50 \%$, T.L.C.- $7500 / \mathrm{mm}^{3}$, ESR-92 $\mathrm{mm}$ at the $1^{\text {st }}$ hour. Liver Functions Tests were within the normal limits. Hepatitis Profile A,B,CNegative. $X$ Ray Chest and Abdomen were normal. U.S.G. showed thickened gall bladder and hepatomegaly with coarse echo texture (Fig. 1). A contrast CT scan was abandoned due to sensitivity of the patient to contrast medium. 


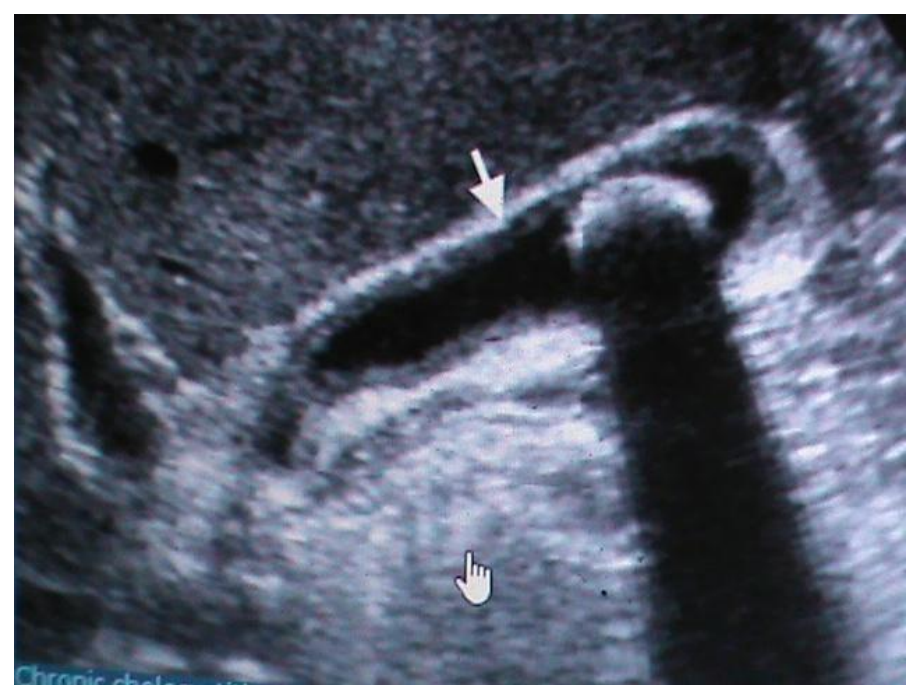

Fig.1

An exploratory Laparotomy was planned. Abdomen was opened by upper right paramedian incision. Visceras were examined and found to be normal. The right lobe of liver was macroscopically pale with whitish appearance. Lower part of the right lobe was enlarged and granular. The gall bladder was normal. A wedge biopsy was taken from the enlarged part of the right lobe of liver. Bleeding from the liver was controlled by gel foam packing. The abdomen was closed in layers.

Histology confirmed the lesion as hepatic granuloma with central caseating necrosis, suggestive of hepatic tuberculosis (Fig 2).

The patient was treated with 3 drug antitubercular regime comprising of isonex, rifampicin \& ethambutol daily for 2 months followed by isonex \& rifampicin daily for the next 7 months. After one month of treatment, the patient showed marked improvement and the patient reported full recovery after completion of the course.

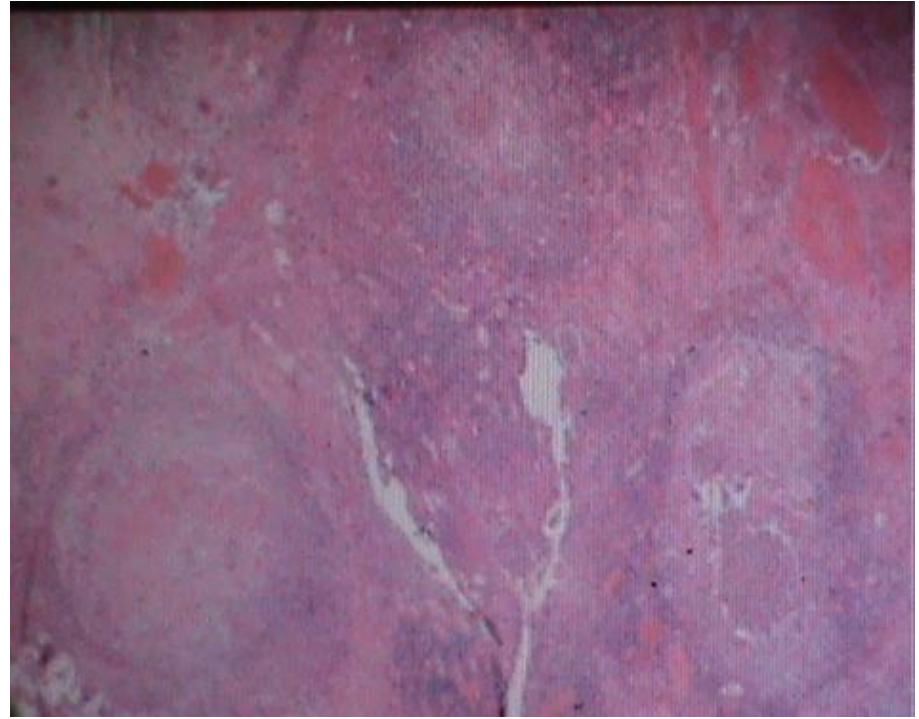

Fig. 2: Granulomatous lesion with central necrosis in liver parenchyma.

\section{Discussion}

Tuberculosis of the liver is a rare entity and a conclusive diagnosis is possible only by histology. Hersch, reported a case of tuberculosis of liver. ${ }^{[5]}$

Mehrotra (1986) discuss a case of tuberculosis of liver which was diagnosed by liver biopsy only. ${ }^{[6]}$ Case of liver tuberculosis have been reported by Terry(1957) ${ }^{[7]}$, and Essop A R (1984) ${ }^{[1]}$

Mamun et al (2011) did culture of liver tissue for mycobacterium tuberculosis for confirmation of diagnosis. $^{[8]}$

Bangro A.K. (2005) reported isolated hepatic tuberculosis $^{[9]}$

If the patient of hepatomegaly does not show improvement on medical treatment the diagnosis should be confirmed by liver biopsy.

\section{Summary}

A case of tuberculosis of liver in 41 year old man who had under gone anti amoebic treatment as reported. And this case was come out as tuberculosis of liver after liver biopsy. The patient was fully recovered after antitubercular treatment. In conclusion unexplained hepatomegaly with normal investigation reports the liver biopsy should be performed for tuberculosis of the liver. 


\section{Acknowledgement}

I am very gratefull to Mr. Kuldeep patidar, Medicos, R.D. Gardi Medical College, Ujjain for typing this article.

\section{References}

1. Essop A R, Posen, JA,Hodkinson, JH,Segal I, Tuberculosis hepatitis ,--a clinical review of 96 cases, Quart J.Med 1984, S3,465

2. Leader S.A. Tuberculosis of liver and gall bladder with abscess formation: a review and case report. -Ann Intern Med. 1952; 37,594

3. Johri B.S. ,kane M P Mudbhat kal N S Isolated tuberculosis of liver Indian $\mathrm{J}$. Medical Sciences 1970,24,16

4. Puri A S, Nayyar A K, Vij J.C. Hepatic tuberculosis Ind .J .Tub 1994;4:131-4

5. Hersch,C.Tuberculosis of liver;South African med J. 1964;38:857

6. Mehrotra J.C. ,Khosla S N ,Chhabra U K .Liver in abdominal tuberculosis, J.Assoc.Phys.India 1986;34;501

7. Terry R.B. Gunnar R.M. Primary military tuberculosis of the liver JAMA 1957:164 ;150

8. Mamun A L Mahatab, Salimar RahmanMohammad Kamal - a case report of hepatic tuberculosis 10.5005/J.P. Journal 10018-1008 Euro-Asian Journal of Hepato-Gastro-Enterology January to June 2011;1(1):35-37

9. Bangro A.K.,Malhotra A S, Isolated Hapetic Tuberculosis, J.India Assoc Paed Surgery 2005:10:105-107. 